\title{
TRATAMIENTO PSICOLÓGICO DEL HÁBITO DE FUMAR: COMPARACIÓN DE DOS MÉTODOS DE REDUCCIÓN GRADUAL
}

\author{
Andrós López de la Llave' , José Maria Bucota² \\ y Marla del Carmen Pórez-Llantada² \\ ' Universidad Europea de Madrid, CEES. \\ 2 Universidad Necional de Educación a Distancia
}

\begin{abstract}
RESUMEN
Cuarenta y dos fumadores fueron sometidos a un programa de tratamiento psicológico para dejar de fumar de forma gradual. Veintidós de ellos, fueron tratados mediante un método basado en la descomposición de los mecanismos psicológicos responsables del mantenimiento del hábito de fumar, y la preparación de los fumadores para la abstinencia (grupo DHF), y los restantes veinte participantes, mediante un método más tradicional, basado en el establecimiento de objetivos progresivos de reducción del consumo (grupo RGT). Los resultados mostraron la superioridad del tratamiento DHF, con un porcentaje mayor de sujetos abstinentes al finalizar el tratamiento y en los seguimientos realizados a los tres, seis y doce meses. Ambos métodos fueron eficaces para lograr reducir el consumo de cigarrillos de los sujetos que no lograron eliminar el hábito. En la discusión se plantea la contribución de estos resultados en el contexto del tratamiento psicológico para dejar de fumar.
\end{abstract}

Palabras clevo: Dejar de Fumar, tratamiento psicológico, reducción gradual.

\begin{abstract}
Forty two smokers were exposed to psychological treatment for the gradual elimination of smoking. Twenty two of them, were treated using a method based both on debilitating psychological mechanisms considered responsible of the maintenance of this habit, and the preparation of smokers for abstinence (group DHF), and the other twenty subjects, were treated through a more traditional method, based on setting progressive goals related to reduction (group RGT). Results showed the superionity of the treatment DHF, with a higher percentage of abstinent subjects at the end of treatment and at followup three, six and twelve months later. Both methods were efficient to reduce the rate of cigarettes smoked per day, in subjects who did not manage to eliminate smoking. The results are discussed in the context of their contribution to the psychological treatment of smoking.
\end{abstract}

Koy Words: Smoking cessation, psychological treatment, gradual elimination.

Correspondencia: Andrés Lopez de la Llave, Departamento de Psicologla, Facultad de Clencias de la Salud, Universidad Europea de Madrid, 28670 Villaviciosa de Odón, Madrid. 


\section{INTRODUCCIÓN}

El consumo de tabaco perjudica gravemente la salud de los fumadores y las personas que los rodean. Fumar deteriora el aparato respiratorio y aumenta el riesgo de padecer infecciones respiratorias, enfermedades cardiovasculares y algunos tipos de cáncer, y por tanto, se considera un hábito nocivo que puede repercutir en la calidad y el tiempo de vida.

Muchos fumadores que desean abandonar el hábito, son incapaces de conseguirlo sin ayuda especializada, y para responder a esta necesidad, han surgido tratamientos de distinto tipo; entre ellos, como demuestran diversos trabajos recopilados por Becoña (1987) y la extensa revisión realizada por Schwartz (1987). programas de intervención psicológica con múltiples estrategias, que sugieren la conveniencia de tratamientos multicomponente en los que se combinen distintas técnicas. En lineas generales, estos programas suelen tener dos grandes objetivos: que se produzca la eliminación de la conducta, y que se mantenga la abstinencia. En numerosos casos, las técnicas psicológicas han resultado eficaces respecto al primer objetivo, pero en menor medida respecto al segundo, planteándose el problema de un elevado índice de recaídas.

El objetivo de la eliminación del consumo, puede intentar lograrse mediante la cesación brusca o la reducción gradual del número de cigarrillos (Günther, Gritsch y Meise, 1992). Aunque la cesación brusca del consumo ha resultado eficaz en algunos casos, determina la aparición del síndrome de abstinencia a la nicotina, cuyos sintomas se presentan dentro de las veinticuatro horas siguientes al cese $y$ se prolongan durante varios dias (APA, 1995); y además, suele realizarse sin que los fumadores estén lo suficientemente preparados para manejar situaciones de riesgo de recaidas, tales como la ansiedad relacionada con el deseo de fumar, o la presión social, que están presentes en numerosas ocasiones. Asi, la experiencia de la abstinencia mediante la cesación brusca, resulta muy aversiva para muchos fumadores que, tarde o temprano, escapan de ella recuperando el hábito de fumar. Para éstos y otros fumadores que ni siquiera lo han intentado, la cesación brusca constituye una situación muy estresante que favorece la evitación de este tipo de tratamientos, surgiendo como alternativa mejor aceptada, los programas que persi- guen la reducción gradual del número de cigarrillos. Estos programas, además de mejorar la motivación de los fumadores hacia el tratamiento, parecen aliviar los síntomas aversivos que provoca la abstinencia, al propiciar que los fumadores se preparen, física y psicológicamente, de manera progresiva, para la situación estresante de abandonar el tabaco por completo.

Los programas de reducción gradual más tradicionales, incluyen diversas técnicas (contratos de contingencias, auto-registros, cambios de marca de cigarrillos, establecimiento de objetivos, reforzamiento social o material, etc.) que pretenden ayudar a los fumadores a reducir el consumo de manera progresiva. El énfasis de estos programas, se sitúa en que el fumador, cada semana u otro periodo de tiempo, consuma menos cigarrillos que en el periodo anterior, y asi, sucesivamente, hasta llegar al objetivo final de la abstinencia. En estos programas, no es trascendente qué cigarrillos se eliminan o cómo se eliminan, ni tampoco, generalmente, si el fumador está preparado para el abandono definitivo; sino que el fumador reduzca el consumo, indiscriminadamente, según los objetivos establecidos, y que, siguiendo esta progresión, abandone la conducta de fumar cuando sea éste el objetivo que corresponda.

Según la revisión de Schwartz (1987), este método ha mostrado una eficacia muy variada (entre el $7 \%$ y el $46 \%$ ) al año de seguimiento; probablemente, porque junto a los aspectos positivos que presenta, como son la reducción gradual y la utilización de técnicas muy útiles, minimiza la importancia de los mecanismos psicológicos que suelen contribuir, poderosamente, al mantenimiento de las conductas habituales; en concreto: la relación funcional entre la conducta y sus antecedentes, y la conducta y sus consecuencias; y las cogniciones subyacentes (creencias, actitudes, etc.) que a través de diversos procesos pueden afectar a la conducta. Así, podría ocurrir, que aún habiendo abandonado el fumador la conducta de fumar, determinados estimulos antecedentes continuasen ejerciendo una notable influencia sobre la probabilidad de aparición de la conducta, o que algunas creencias rigidas y poderosas, estuviesen afectando, perjudicialmente, la percepción e interpretación subjetivas de los sintomas de abstinencia, en cuyo caso existirla un riesgo de recaida muy alto. 
Para intentar subsanar este problema, Buceta y Thomas (1983) emplearon un método de tratamiento de reducción gradual, en el que el objetivo no era la disminución indiscriminada del consumo de cigarrillos, sino la descomposición del hábito mediante el debilitamiento de las relaciones funcionales entre antecedentes, conducta y consecuencias, y la intervención sobre cogniciones perjudiciales; debiendo producirse la reducción del consumo como consecuencia de este proceso de descomposición.
Buceta y López de la Llave (1989) adaptando el programa de Buceta y Thomas (1983) realizaron un estudio en el que participaron 35 sujetos. La media de consumo de tabaco estimado por los sujetos antes del tratamiento. fue de 22.07 cigarrillos al dia y el consumo observado en la linea base mediante autoregistros dlarios durante una semana, fue de 18.22 cigarrillos. El programa inclula entre sus componentes técnicas como: los auto-registros, el control de estimulos, la prevención de la respuesta, y el entrenamiento en habilida-

\section{Tabla 1.- Supuestos bésicos del tratamiento (Buceta y López de la Llave, 1989)}

$1^{\circ}$. El comportamiento de fumar es un habito aprendido, fuertemente consolidedo en los fumadores y diflcil de eliminar, por lo que aquellos que deseen cesar en ol hablto y mantenerse abstinentes, necealtaran dedicar tiempo y esfuerzo a conseguito (Shifiman, Read, Maltese, Rapkin y Jarvik, 1985).

$2^{\circ}$. El comportamiento de fumar se produce en situaciones especificas, determinadas por las condiciones antecedentes y consecuentes de la respuesta, to que implica la necesidad del antisis funclonal del comportamiento, como paso previo a la puesta en marcha de estrateglas para la eliminación del mismo. Asimiamo, ef comportamiento de fumar puede estar favorecido por pensamientos y/o creencias que aumentan la disposición del fumador a llevar a cabo ta conducta y que, por lo tanto, tambien deben ser considerados en el análisis funcional.

$3^{\circ}$. El comportamiento de fumar puede eliminarse cuando: (a) se debilita el vinculo existente entre los estimulos antecedentes en cuya presencia tiene lugar la conducta de manera habitual y la propia conducta, pudiendo facilitar este proceso ol vincular tales estimulos a conductas alternativas incompatibles a la conducta de fumar; (b) $s e$ modifican pensamientos concretos y creencies estables que favorecen la disposición dol fumador hacia la conducta de fumar, y se desarrollan otros que aumentan su disposición a la abstinencia; y (c) se elimina el cartacter reforzante de ta conducta de fumar.

$4^{\circ}$. Fumar produce efectos fislologicos en el organismo, tales como tolerancia y dependencia, por lo que cesar bruscamente en el consumo de tabaco, puede hacer aparecer el sindrome de abstinencia a la nicotina. La reducción gradual del consumo elimina este problema; sin embergo, fumar un número reducido de cigarrillos puede resultar altamente reforzante, lo que podria contribuir poderosamente al fortalecimiento del hábito, vinculándose la conducta de fumar a consecuenclas gratificantes y estimulos antecedentes determinados.

$5^{\circ}$. Teniendo en cuenta el apartado anterior, parece conveniente que un programa de intervención comience con un proceso de reducción gradual, pero sin permitir que se dilate en el tiempo el perlodo de bajo consumo de cigarrillos. Asl, podrian considerarse dos etapas, una primera en que se intentarfa lograr esa reducción (gradual) del consumo, y una segunda en que se deberia buscar el cese total de la conducta y el mantenimiento permanente de la abstinencia.

$6^{\circ}$. El procedimiento de intervención deberla centrarse en el debilitamiento de los vinculos entre los antecedentes, la conducta de fumar y las consecuencias, en la modificación de pensamientos y creencias perjudiciales y en el entrenamiento de los sujetos en habilidades que les permitan mantenerse abstinentes. Dejar de fumar y mantenerse sin fumar son dos cosas diferentes y conviene tener ambas en cuenta, la segunda como objetivo central de la intervención y la primera como paso previo.

$7^{\circ}$. En la linea utilizada por Buceta y. Thomas (1983) y Buceta y López de la Llave (1989), con vistas a incrementar la probabilidad de exito, en el momento de dar el paso decisivo a la ceseción total, parece conveniente que se establezcan "periodos de prueba" que disminuyan el temor de los fumadores, a menudo habitual, a dar este paso tan importante, facilitando, asimismo, la oportunided de recoger información respecto a las dificultades que el fumador se va a encontrar, cuando deje de fumar, para mantener la abstinencia. Estos "periodos de prueba" deberlan programarse cuidadosamente, de forma que el fumador tuviera exito en el intento y se fortaleciera su auto-confianza.

$8^{\circ}$. La intervención sobre el comportamiento de fumar requiere la participación activa de los usuarios desde el primer momento. Se deberla fomentar esta participeción tanto en los niveles formales (horarios, lugares...), como procesuales (toma de decisiones, aceptación de las tareas...), de forma que la intervención pudiera ser verdaderamente útil. Además, el desarrollo de atribuciones internas en relación con los exitos que se vayan alcenzando, aumentarla la percepción de auto-dominio y auto-eficacia de los sujetos, lo que a su vez consolidarla en mayor medida los logros terapeuticos. 
des diversas, como la relajación, las auto-instrucciones, las habilidades sociales y otras habilidades de afrontamiento según las necesidades de cada sujeto; además, se contemplaba la posibilidad de utilizar la técnica de fumar rapido y diversas estrategias cognitivas. Las principales caracteristicas del programa, sin embargo, consistian en el papel activo de los sujetos, en el trabajo sobre sus expectativas en cuanto al programa y a su exigencia, y en el proceso de reducción gradual del consumo de cigarrillos como resultado de la puesta en práctica de las distintas estrategias; y no como objetivo del programa con independencia del proceso. Básicamente, el objetivo de las estrategias consistia en descomponer el hábito de fumar debilitando los vinculos existentes entre estimulos, respuesta y consecuencias, modificando cogniciones relevantes y entrenando a los fumadores en técnicas que les sirvieran para enfrentarse con exito, antes y después de la abstinencia, a las situaciones de riesgo relacionadas con la conducta de fumar. Se partia del supuesto, de que la descomposición del habito, junto al fortalecimiento de la inmunidad de los sujetos mediante la mejora de sus recursos, deberian ser responsables de los beneficios terapéuticos a corto y a largo plazo. Finalizaron el programa 26 de los 35 sujetos que lo iniciaron ; las tasas de abstinencia, para estos 26 sujetos, al final del tratamiento, al mes y a los seis meses de seguimiento, fueron del $84,62 \%$, del $73,07 \%$ y del $53,84 \%$ respectivamente, indicando que este tipo de programa puede resultar muy eficaz. Los supuestos en los que se basa este tratamiento, pueden encontrarse resumidos en la tabla 1.

La prometedora perspectiva del programa utilizado por Buceta y Lopez de la Llave (1989), plantes la conveniencia de continuar investigando en esta dirección, con estudios que aporten más datos sobre la eficacia del tratemiento de reducción gradual basedo en la descomposición del hábito de fumar y la preparación de los fumadores para afrontar con éxito el reto de la abstinencia. En este contexto, el proposito de la presente investigación, consiste en comparar este tipo de tratamiento, con otro de reducción gradual pero de corte tradicional, es decir, basado en la disminución progresiva e indiscriminada del consumo de cigarrillos.

\section{MÉTODO}

Sujetos

A través de carteles, folletos y contactos directos en lugares públicos, un centro de salud y diversas empresas, se ofreció la posibilidad de participar en un programa para dejar de fumar. Entre las personas que respondieron, 42 sujetos, 19 mujeres y 23 varones, con edades comprendidas entre los 26 y los 60 años (Media=40,33; DT=8,03), participaron en este estudio. La media de ańos fumando era de 21,32 (DT = 8.6), y el número de cigarrillos consumidos diariamente, según la estimación global de los sujetos, oscilaba entre 5 y 60 cigarrillos (Media=26,66 cigarrillos al dia, DT=11,8). El $82 \%$ de los sujetos habia intentado, previamente, dejar de fumar.

\section{Matoriales}

Cuestionario previo para el programa de dejar de fumar. Este cuestionario fue elaborado para recoger la siguiente información de los sujetos: (a) sus características personales y sociales (nombre, edad, sexo, estado civil y profesión); (b) el consumo estimado de cigarrillos; (c) la persistencia del hábito; (d) la realización de intentos anteriores para dejar de fumar (si se hablan producido y duración de los mismos); (e) la auto-evaluación de los sujetos sobre las ganas que tenian de dejar de fumar, la expectativa del esfuerzo que tendrian que realizar para lograr este propósito y la ayuda que crelan necesiter de los demás (familiares, companeros, terapeuta) para dejar de fumar; y (f) el deseo/necesided de fumar (mediante algunos ftems del cuestionario "Tolerancia a la nicotina" de Fagerstróm, 1978).

Hojas de Auto-rogistros. Se elaboraron ejemplares estandarizados para el auto-registro de datos relacionados con el consumo de cada cigarrillo durante los periodos intersesiones. Les hojas de auto-registro tenian impresas cinco columnas: (1) la hora; (2) el lugar; (3) la compania, que incluia las categorlas de fumar estando solo o en companila de alguna persona; (4) la actividad que se estaba realizando mientras se fumaba; y (5) el grado de placer que habia producido consumir cada cigarrillo, valorado en una escala subjetiva de 1 a 10 puntos.

Folleto Lecturas sobre el tabaco. El folleto Lecturas sobre el tabaco es la adaptación de una parte del texto El tabaco y su abandono, 
de Becoña, Galego y Lorenzo (1988). El contenido de este cuaderno hacia referencia a los siguientes temas: (a) Una breve historia del tabaco; (b) Los tipos de tabacos; (c) Los compuestos del tabaco: La nicotina y el monóxido de carbono; (d) La salud y el consumo de tabaco: enfermedades influidas por el consumo de tabaco.

EC50 Micro Smokentyzer. Con la finalidad de disponer de una medida objetiva de la abstinencia y del proceso de reducción gradual, se em-plé́ un analizador de monóxido de carbono (CO) en el aire expirado. Esta variable se evaluaba tomando muestras, del aire expirado por el fumador, en el EC50 Micro Smokerlyzer Portable.

Carbon Monoxide Monitor (Bedfont Technical Instruments Ltd, Sittingboume, Kent, U.K.). Mediante este aparato se obtiene una medida de partes por millón (ppm) de monóxido de carbono en el aire expirado.

\section{Control de variables intervinientes}

Con el objeto de controlar su posible influencia en los resultados del estudio, se consideraron las siguientes variables intervinientes:

1. Variables sociodemograficas. (1) Edad; (2) sexo; (3) la existencia de algún familiar, actualmente, fumador; $y$, si es asl, (4) el hecho de que fume cigarrillos de la misma marca.

2. Variables en relación con la evolución del hábito. (1) La persistencia del hábito, medida en años como fumador habitual, según los informes del propio fumador; y (2) la duración máxima de los periodos de abstinencia previos, medido en semanas, también según los informes de los sujetos.

3. Variables mothacionales. (1) Las ganas de dejar de fumar con la que los sujetos inician el tratamiento; (2) la ayuda que cada fumador cree necesitar de familiares y otras personas para ser capaz de abandonar el hábito; (3) el esfuerzo que consideraba que serla necesario realizar para dejar de fumar (la medida de estas tres variables se obtenia mediante la auto-evaluación de los sujetos en una escala de 0 a 10 puntos); (4) el estar dispuesto a pagar dinero para dejar de fumar; y (5) en el caso de una contestación afirmativa a la anterior cuestión, qué porcentaje de su sueldo mensual estaria dispuesto a pagar.

4. Variables relativas al uso y dependencia del tabaco. (1) Consumo estimado: teniendo en cuenta el número de cigarrillos que, por término medio, estimaba consumir al dia cada sujeto, antes de iniciarse el tratamiento; y (2) deseo/necesidad de fumar: considerando la medida que se obtenía en esta variable, de la suma de puntuaciones a los cuatro items, del Cuestionario de tolerancia a la nicotina de Fagerstrom (1978), que se habian incluido en el cuestionario previo para dejar de fumar.

\section{Variables dependientes}

Para observar la eficacia del tratamiento fueron contempladas las siguientes variables dependientes:

1. Número de sujetos abstinentes al finalizar la intervencion, determinando su estatus mediante los auto-registros y el analizador de CO (se considero que menos de 10 ppm de CO correspondla a un no fumador); número de sujetos abstinentes en los seguimientos a los tres y seis meses, mediante informes globales de los sujetos; y número de sujetos abstinentes on el seguimiento al ano, nuevamente, contrastado su informe con una medida de $\mathrm{CO}$.

2. Número de cigarrillos consumidos al dia al final del tratamiento, según los auto-registros de los sujetos e informes globales en cada seguimiento.

3. Número de semanas, durante los doce meses siguientes al final del tratamiento, en los que los sujetos que finalizaron el tratamiento se hablan abstenido de fumar; obteniéndose esta medida de la información proporcionada por los propios sujetos, en los contactos periodicos mantenidos durante el seguimiento.

4. Nivel de CO contenido en el aire expirado, al final del tratamiento y en el seguimiento realizado a los 12 meses.

\section{Variables independlentes}

Las variables independientes fueron los dos tipos de tratamiento: (a) Tratamiento de reducción gradual tradicional (RGT) y (b) Tratamiento de descomposición del hábito de fumar $y$ entrenamiento en habilidades de afrontamiento (DHF). En las tablas 2 y 3, se describen, respectivamente las caracteristicas de estos tratamientos, y en la tabla 4 se detalla el procedimiento empleado en cada uno de ellos, pudiendo encontrarse una explicación más amplia del tratamiento DHF en el escrito de Lopez de la Llave y Buceta Tratamiento psicológico del hábito de fumar (1996). 
Tabla 2.- Fases del tratamiento de Reducción gradual tradicional (RGT)

1* Fase:

ENTREVISTA INICIAL, EVALUACIONY LINEA BASE

2* Fase:

TRATAMENTO

3* Fase:

MANTENMMENTO
OB.JETNOS:

-Introducción al programa.

-Establecimiento de la linea

base de consumo de cigarrillos

DURACION:

Contacto inicial individual y

1 Sesión
ACTMIDADES:

-Presentación del programa como un proceso para dejar de fumar basado en eliminar las consecuencias negativas asociadas al abandono, mediante la reducción gradual de la ingesta por medio de la disminución en el consumo de cigarrillos y/o la reducción de la cantidad de cada cigarrillo que se consume.

-Explicación y adiestramiento en el uso de los autorregistros.

-Elaboración, y discusión, de listas de ventajas - inconvenientes de dejar de fumar.

- Medida del nivel de monoxido de carbono en el aire expirado

\section{ACTMDADES:}

-Establecimiento del plan de reducción. -Registro y grafico de la conducta de fumar -Ejerciclos de control de astimulos y de prevención de la respuesta.

- Actividades para reducir el consumo de cigarrillos y el caracter reforzante de fumar:

- Retraso de la conducta.

- Ensayos de retención del humo.

- Reducción de nicotina y alquitrán.

-Medida del nivel de monóxido de carbono.

\section{ACTMDADES:}

-Anticipacion de situaciones con alta probabilidad de fumar.

-Entrenamiento en habilidades para afrontar situaciones de resgo:

. Relajación.

DURACKON:

Asertividad.

2 Sesiones y la posibilidad de contactos telefónicos después de finalizada la intervención 
Tabla 3.- Caracteristicas del tratamiento de Descomposición del hábito de fumar y entrenamiento en habilidades de afrontamiento (DHF)

$1^{\circ}$ Fase:

INTRODUCCIONY RECOGIDA DE DATOS

2 Fase:

DESCOMPOSICIONNDLL HABITO DE FUMAR MEDIANTE EL DEBILITAMIENTO DE LOS VINCULOS $E \leftrightarrow R \leftrightarrow C$, ENTRENAMIENTOEN HABILIDADES DE COPING DIVERSAS

3* Fase:

PRUEBAS DE CESACION Y ABSTINENCIA.

4* Fase:

MANTENIMIENTO

DE LA ABSTINENCIA
OQJETMOS:

-Presentación del programa -Ajuste de expectativas.

-Establecimiento de la línea base.

\section{DURACIÓN}

Contacto inicial individual y dos sesiones presenciales

OBJETNOS:

-Análisis funcional de la conducta de fumar.

-Debilitamiento de los vinculos estimulo $\leftrightarrow$ conducta $\leftrightarrow$ consecuencia -Entrenamiento y adquisición de habilidades de coping.

\section{DURACION}

4 sesiones presenciales

OBJETINOS:

-Realización de periodos de abstinencia a prueba.

-Inicio de la abstinencia continuada.

\section{DURACION}

1 sesion

\section{ACTIVIDADES}

-Explicación de los objetivos de la intervención. -Descripción del nivel de exigencia del programa. -Explicación del papel activo de los participantes. -Explicación básica de los mecanismos que man tienen la conducta de fumar (antecedentes $\leftrightarrow$ conducta $\leftrightarrow$ consecuencias).

- Adiestramiento en el uso de los auto-registros.

- Medida de CO en el aire expirado.

- Elaboración de listas de ventajas e inconvenientes de dejar de fumar.

\section{ACTIVIDADES:}

-Registro y gráfico de la conducta de fumar. -Ejercicios de control de estímulos.

-Prevención de respuesta.

-Actividades para reducir el caracter reforzante de la conducta de fumar:

Retraso de la conducta.

.Cepillado de los dientes con pasta dental antes y después de fumar

- Medida de CO en el aire expirado

\section{ACTMIDADES:}

-Negociación del momento y duración de los periodos de prueba.

-Prevención de dificultades y ensayo de estrategias para controlarlas

-Análisis de las dificultades encontradas durante los periodos de abstinencia y búsqueda de soluciones

-Refuerzo de los logros conseguidos durante los periodos de abstinencia

\section{ACTIVIDADES:}

-Anticipación de situaciones de riesgo con mayor probabilidad de fumar.

-Ensayo de estrategias para controlar las situaciones de riesgo

-Anticipación de consecuencias de vivir sinfumar.
DURACION

1 sesion
- Mantenerse sin fumar posibles recaidas. 
Tabla 4.- Procedimiento empleado al aplicar cada programa de tratamiento (1/5)

\begin{tabular}{|c|c|c|}
\hline 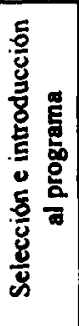 & $\begin{array}{c}\text { Grupo } \\
\text { s } \\
\\
\text { DHF } \\
\text { y } \\
\text { RGT }\end{array}$ & $\begin{array}{l}\text { ACTIVIDADES: Cada sujeto fue entrevistado en el lugar donde luego se desarrollaría el } \\
\text { programa ; se les entregó el Cuestionario previo para el programa de dejar de fumar, y } \\
\text { ejemplares de auto-registro ; se comentó cómo cumplimentar los instrumentos. Se fijó la fecha } \\
\text { de la } 1^{2} \text { entrevista con el terapeuta y la fecha de la } 1^{2} \text { sesión del programa. } \\
\text { Tareas intersesiones: Cumplimentar el Cuestionario, y registro de la conducta de fumar } \\
\text { durante los } 7 \text { días previos al inicio del programa. }\end{array}$ \\
\hline
\end{tabular}

\begin{tabular}{|c|c|c|}
\hline 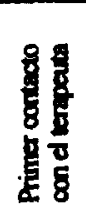 & $\begin{array}{l}\text { Grupo } \\
\text { s } \\
\text { DHF y } \\
\text { RGT }\end{array}$ & $\begin{array}{l}\text { Reunión del terapeuta con cada uno de los sujetos, en el lugar donde luego se llevaria a cabo } \\
\text { todo el programa de tratamiento. } \\
\text { Actividades: Resolución de dudas respecto a la cumplimentación de cuestionarios y/o } \\
\text { autorregistros. Se tomó la primera medida de } \mathrm{CO}\end{array}$ \\
\hline & & \\
\hline
\end{tabular}

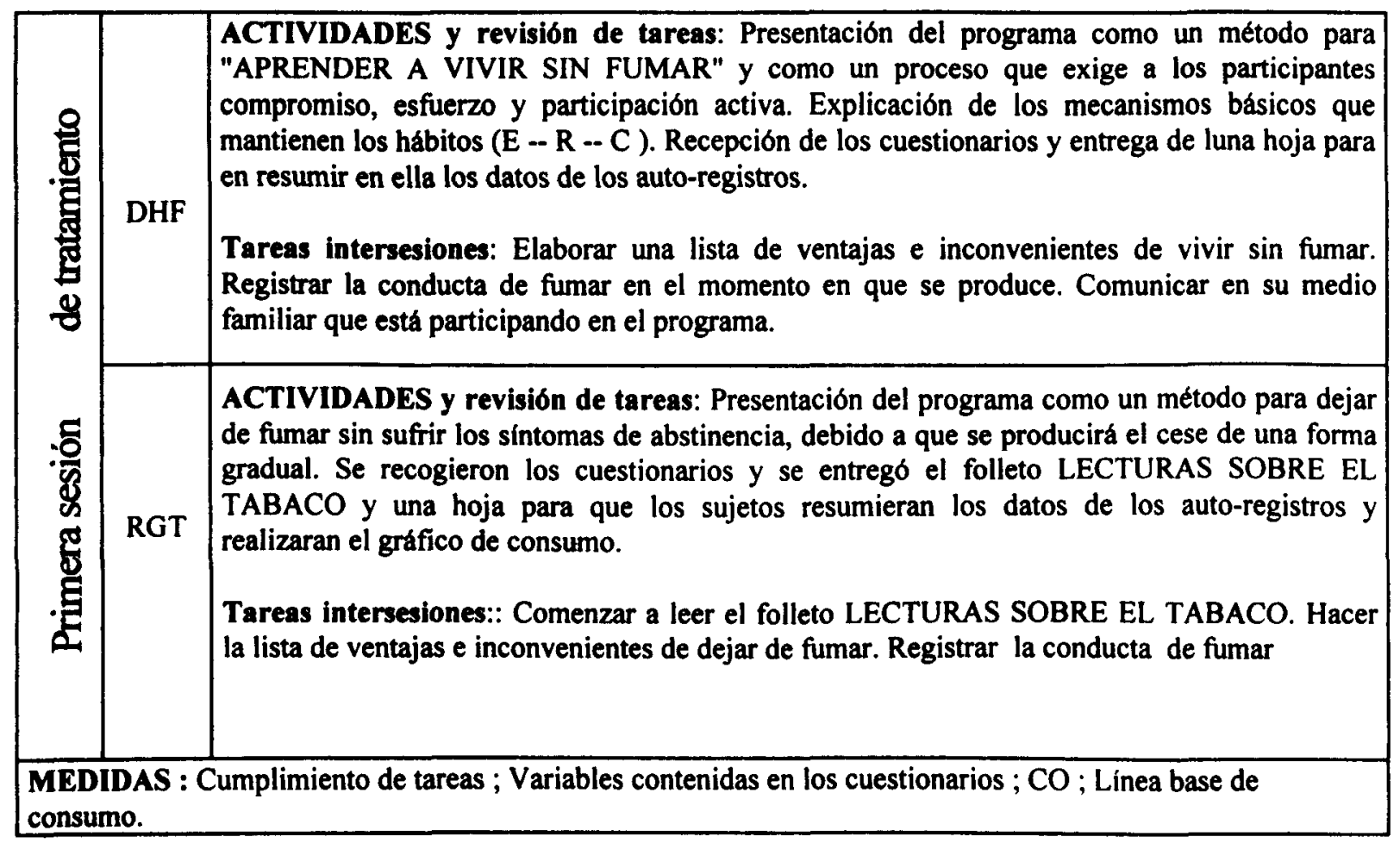


Tabla 4.- Procedimiento empleado al aplicar cada programa de tratamiento (2/5)

\begin{tabular}{|c|c|c|}
\hline 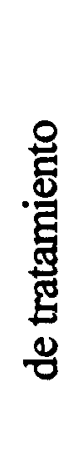 & DHF & $\begin{array}{l}\text { Revisión de tareas: Los sujetos se autoevaluaron en cuanto al grado de cumplimiento de las } \\
\text { actividades encomendadas en la anterior sesión de tratamiento. Se les entregó la Hoja para el } \\
\text { Registro de Datos Individuales para reflejar en ella las anotaciones de los autorregistros. } \\
\text { Actividades: Se reforzó a los sujetos por la realización de las tareas. Se comentó la filosofia } \\
\text { del programa y se explican los fundamentos del análisis funcional de la conducta. Se entregó a } \\
\text { los sujetos el folleto LECTURAS SOBRE EL TABACO } \\
\text { Tareas intersesiones: Registro del consumo de cigarrillos en los autorregistros. Explicar a } \\
\text { familiares y amigos en qué consiste el programa y comentar la lista de ventajas e } \\
\text { inconvenientes. }\end{array}$ \\
\hline 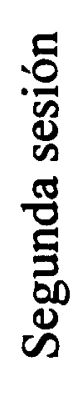 & RGT & $\begin{array}{l}\text { Revisión de tareas: Se entregó de la Hoja para el Registro de Datos Individuales para llevar a } \\
\text { la hoja los datos de los autorregistros realizando el gráfico de consumo y para la } \\
\text { autoevaluación en el cumplimiento de tareas. } \\
\text { Actividades: Comentarios sobre contenido del folleto LECTURAS SOBRE EL TABACO. Se } \\
\text { comentan las razones para dejar de fumar. Se mide el CO. } \\
\text { Tareas intersesiones:: Registrar la conducta de fumar. Comunicar a familiares y amigos que } \\
\text { se está participando en este programa. Reducir el consumo en al menos un } 15 \% \text { ( } 3 \text { cigarrillos } \\
\text { de cada } 20 \text { ). }\end{array}$ \\
\hline
\end{tabular}

\begin{tabular}{|c|c|c|}
\hline 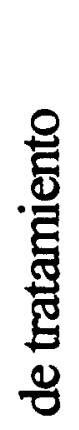 & DHF & $\begin{array}{l}\text { REVISIÓN DE TAREAS: Análisis de autorregistros y representación en Hoja para el } \\
\text { Registro de Datos Individuales. Autoevaluación del cumplimiento de las actividades } \\
\text { intersesiones. Refuerzo a la realización de tareas. } \\
\text { ACTIVIDADES: Se explicó cómo proceder al estudio de los autorregistros para jerarquizar } \\
\text { las situaciones de consumo e identificar las de más alta probabilidad de fumar. Realización de } \\
\text { las jerarquías. Ejercicios de prevención de respuesta. Medida de CO y comentarios. } \\
\text { Tareas intersesiones: Autorregistros. Abstenerse de fumar en la situación escogida y pactada } \\
\text { con el terapeuta }\end{array}$ \\
\hline 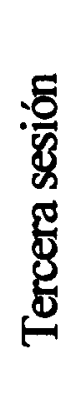 & RGT & $\begin{array}{l}\text { REVISIÓN DE TAREAS: Los sujetos llevaron los datos de los registros a la Hoja para el } \\
\text { Registro de Datos Individuales y se autoevaluaron en cuanto al cumplimiento de los } \\
\text { compromisos de reducción de su consumo, y se reforzó por esa reducción. } \\
\text { ACTIVIDADES: Se comentaban los procedimientos utilizados para fumar menos y su } \\
\text { eficacia. Se explicaba la posibilidad de cambios de marcas de tabaco. Se medía el monóxido } \\
\text { de carbono y se comentaban los resultados. } \\
\text { Tareas intersesiones: Reducción del consumo en, al menos un } 25 \% \text { sobre la línea base. } \\
\text { Registrar el consumo }\end{array}$ \\
\hline
\end{tabular}


Tabla 4.- Procedimiento empleado al aplicar cada programa de tratamiento (3/5)

\begin{tabular}{|c|c|c|}
\hline 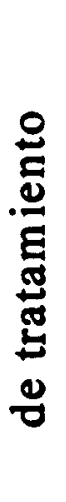 & $\mathrm{HF}$ & $\begin{array}{l}\text { Revisión de tareas: Análisis de los autorregistros, evaluación del cumplimiento de } \\
\text { actividades asignadas y traslado de los datos a la Hoja para el Registro de Datos } \\
\text { Individuales. } \\
\text { Actividades: Se reforzó a los sujetos por el cumplimiento de las tareas y se hicieron } \\
\text { comentarios sobre las dificultades encontradas. Se explicaron procedimientos para reducir el } \\
\text { carácter reforzante de la conducta. Medidas de CO. } \\
\text { Tareas intersesiones: Autorregistros. Abstenerse de fumar en } 1^{2} \text { y } 2^{2} \text { situaciones más } \\
\text { populares determinadas en cada caso por los autorregistros. Realizar ejercicios para reducir } \\
\text { el placer que produce fumar (cepillado de dientes, retrasar el consumo después de comer o } \\
\text { tomar cafe...). }\end{array}$ \\
\hline 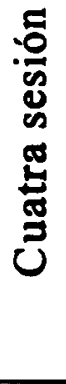 & RGT & $\begin{array}{l}\text { Revisión de tareas: Los sujetos llevaron los datos de los registros a la Hoja para el Registro } \\
\text { de Datos Individuales y se autoevaluaban en cuanto al cumplimiento de los compromisos de } \\
\text { reducción de su consumo. } \\
\text { Actividades: Se explicaba la técnica de retención del humo y se realizaban ensayos de la } \\
\text { misma. Se medía el monóxido de carbono. } \\
\text { Tareas intersesiones: Reducción del consumo en al menos un } 40 \% \text { sobre la línea base. } \\
\text { Registrar el consumo. }\end{array}$ \\
\hline
\end{tabular}

\begin{tabular}{|c|c|c|}
\hline 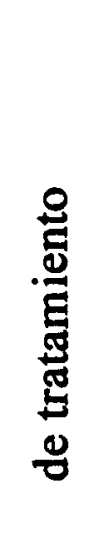 & DHF & $\begin{array}{l}\text { Revisión de tareas: Evaluación del cumplimiento de las tareas. Análisis de los autorregistros } \\
\text { en función de los compromisos adquiridos en la sesión anterior. } \\
\text { Actividades: Se reforzó a los sujetos por el cumplimiento de los ejercicios pactados en la } \\
\text { sesión anterior y se comentaron las dificultades halladas durante la semana. Se realizaron } \\
\text { ejercicios de prevención de respuesta durante la sesión. Se comentó la importancia de los } \\
\text { pensamientos en el mantenimiento de las conductas. Adiestramiento en técnicas de } \\
\text { relajación. Medida de CO. } \\
\text { Tareas intersesiones:: Autorregistros. Lectura de la lista de pensamientos distorsionados e } \\
\text { identificación y registro de los utilizados por cada sujeto. Abstenerse de fumar en situaciones } \\
1^{*}, 2^{\star} \text { y } 3^{\star} \text { y aumentar el intervalo sin fumar en situaciones de alto valor placentero (primer } \\
\text { cigarrillo, tras las comidas). }\end{array}$ \\
\hline 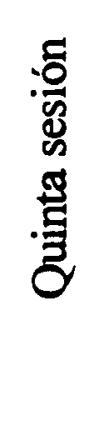 & RGT & $\begin{array}{l}\text { Revisión de tareas: Los sujetos llevaron los datos de los registros a la Hoja para el Registro } \\
\text { de Datos Individuales y se autoevaluaban en cuanto al cumplimiento de los compromisos de } \\
\text { reducción de su consumo. } \\
\text { Actividades: Se comentaban los procedimientos utilizados hasta ahora para fumar menos. Se } \\
\text { comentaban las listas de ventajas e inconvenientes. Se media el monoxido de carbono y se } \\
\text { comentaban, en su caso, los resultados. } \\
\text { Tareas intersesiones:: Reducción del consumo en al menos un } 50 \% \text { sobre la línea base. } \\
\text { Registrar el consumo. }\end{array}$ \\
\hline
\end{tabular}


Tabla 4.- Procedimiento empleado al aplicar cada programa de tratamiento (4/5)

\begin{tabular}{|c|c|c|}
\hline 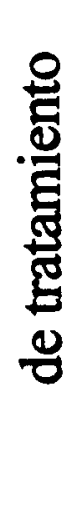 & DHF & $\begin{array}{l}\text { Revisión de tareas: Reflejar el cumplimiento de tareas y los autorregistros en Hoja para el } \\
\text { Registro de Datos Individuales. Análisis de los registros de pensamientos. } \\
\text { Actividades: Ejercicios de racionalización partiendo de los registros de pensamientos. } \\
\text { Prevención de respuesta. Ejercicios de relajación. Explicación del concepto habilidades } \\
\text { sociales con ejercicios. Se hacen comentarios sobre posibles consecuencias del cese } \\
\text { (síntomas de abstinencia, aumento de peso...) Explicación de la actividad CESAR COMO } \\
\text { PRUEBA. Medida del CO. Explicación de la técnica de retención del humo. } \\
\text { Tareas intersesiones: Autorregistros. Aumento de las situaciones donde realizar restricción } \\
\text { de estimulos y/o inicio de pruebas de cesación. Realizar conductas incompatibles } \\
\text { (Relajación, caminar, cambiar de actividad...). }\end{array}$ \\
\hline 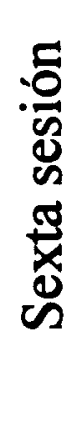 & RGT & $\begin{array}{l}\text { Revisión de tareas: Los sujetos llevaron los datos de los registros a la Hoja para el Registro } \\
\text { de Datos Individuales y se autoevaluaron en cuanto al cumplimiento de los compromisos de } \\
\text { reducción de su consumo. } \\
\text { Actividades: Se comentaron los procedimientos utilizados para fumar menos, y la } \\
\text { importancia del apoyo social en la abstinencia. Se explicó el concepto de autorrefuerzo y la } \\
\text { técnica de retención del humo. Se medía el monóxido de carbono. } \\
\text { Tareas intersesiones: Reducción del consumo en al menos un } 75 \% \text { sobre la línea base. } \\
\text { Registrar el consumo. }\end{array}$ \\
\hline
\end{tabular}

\begin{tabular}{|c|c|c|}
\hline 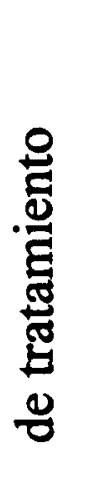 & DHF & $\begin{array}{l}\text { REVISIÓN DE TAREAS: Evaluación del cumplimiento de las tareas. Análisis de los } \\
\text { autorregistros en función de los compromisos adquiridos en la sesión anterior. } \\
\text { ACTIVIDADES: Se reforzó a los sujetos por el cumplimiento de las actividades pactadas en } \\
\text { la sesión anterior y se comentaron las dificultades halladas por aquellos que habían iniciado } \\
\text { pruebas de cesación. Realización de ejercicios de relajación y de prevención de respuesta } \\
\text { durante la sesión. Medida de CO. } \\
\text { TAREAS: Autorregistros de consumo. Pruebas de cesación o, en su caso, continuación de la } \\
\text { abstinencia. Evaluación de situaciones en cuanto al riesgo de fumar y registro de } \\
\text { pensamientos "protectores". }\end{array}$ \\
\hline 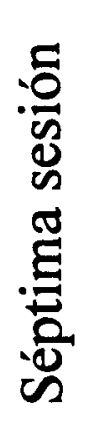 & RGT & $\begin{array}{l}\text { REVISIÓN DE TAREAS: Los sujetos llevaron los datos de los registros a la Hoja para el } \\
\text { Registro de Datos Individuales y se autoevaluaron en cuanto al cumplimiento de los } \\
\text { compromisos de reducción de su consumo. } \\
\text { ACTIVIDADES: Se comentaba la marcha del programa y se reforzaba la abstinencia de los } \\
\text { sujetos que ya no fumaban entregándoles el diploma de no fumador. Se comentaron las } \\
\text { posibles consecuencias del cese (síntomas de abstinencia, aumento de peso...). Se comentó el } \\
\text { posible uso de chicles de nicotina. Se medía el monóxido de carbono. } \\
\text { TAREAS: Reducción del consumo hasta la cesación. Registrar el consumo. }\end{array}$ \\
\hline
\end{tabular}


Tabla 4.- Procedimiento empleado al aplicar cada programa de tratamiento $(5 / 5)$

\begin{tabular}{|c|c|c|}
\hline & DHF & $\begin{array}{l}\text { REVISIÓN DE TAREAS: Se reforzó el cumplimiento de los compromisos y se analizaron } \\
\text { los problemas sugiriendo alternativas. Los sujetos llevaron los datos de los registros a la Hoja } \\
\text { para el Registro de Datos Individuales y evaluaron el cumplimiento de tareas. } \\
\text { ACTIVIDADES: Se explicó cómo el programa seguirá mediante contactos telefónicos y } \\
\text { cuándo recurrir al terapeuta; se comentó la previsibilidad de las situaciones de recaída y } \\
\text { cómo afrontarlas. Se hicieron comentarios sobre posibles consecuencias del cese (síntomas } \\
\text { de abstinencia, aumento de peso...). Se agradeció a los sujetos su participación y se les } \\
\text { entregó un diploma, con la indicación de que lo pusieran en un lugar donde lo vieran a } \\
\text { menudo, para que les recordara su decisión de no fumar. Se comunico a los sujetos la medida } \\
\text { de CO. } \\
\text { TAREAS: Iniciar y/o continuar la abstinencia. Comentar las ventajas de no fumar y constatar } \\
\text { su aparición. }\end{array}$ \\
\hline & RGT & $\begin{array}{l}\text { REVISIÓN DE TAREAS: Los sujetos llevaron los datos de los registros a la Hoja para el } \\
\text { Registro de Datos Individuales y se autoevaluaron en cuanto al cumplimiento de sus } \\
\text { compromisos. } \\
\text { ACTIVIDADES: Se felicitó a los sujetos que habian cesado y se animó a los otros para } \\
\text { cesar; se explico cómo el programa seguiria mediante contactos telefonicos y cuándo recurrir } \\
\text { al terapeuta; se comentó la previsibilidad de las situaciones de recaida y cómo afrontarlas, y } \\
\text { se entregó a todos un diploma en que se mencionaba su participación en el programa. Se } \\
\text { media el monóxido de carbono y se comentaban los resultados. } \\
\text { TAREAS: Abstenerse de fumar. }\end{array}$ \\
\hline
\end{tabular}

\begin{tabular}{|c|c|c|}
\hline 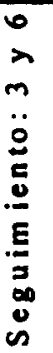 & $\begin{array}{l}\text { Grupos } \\
\text { DHF } \\
\text { y } \\
\text { RGT }\end{array}$ & $\begin{array}{l}\text { Se contactó con cada sujeto, al menos una vez, antes de los tres meses, manteniendose una } \\
\text { conversación sobre la marcha del proceso, haciendo hincapie en la preparación de situaciones } \\
\text { de recalda previsible. Se recordaba a los sujetos las estrategias de control de pensamientos y } \\
\text { los beneficios del apoyo social. } \\
\text { Cuando fueron los sujetos quienes buscaron el contacto se mantuvo la misma línea que en la } \\
\text { intervención, adecuándola a su demanda concreta. } \\
\text { REVISIóN DE TAREAS: Se comentaron con los sujetos las habilidades que habian puesto } \\
\text { en marcha para afrontar la situación de abstinentes. }\end{array}$ \\
\hline
\end{tabular}

\begin{tabular}{|c|c|c|}
\hline 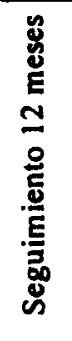 & $\begin{array}{c}\text { Grupo } \\
s \\
\\
\text { DHF } \\
y \\
\text { RGT }\end{array}$ & $\begin{array}{l}\text { En primer lugar se estableció contacto telefónico con cada sujeto para establecer una cita, en } \\
\text { la que se reuniria con los otros miembros de su grupo. } \\
\text { ACTIVIDADES } \\
\text { En esa reunión, en la que en primer lugar se mantuvo una cita individual y después se invitó a } \\
\text { los asistentes a un refresco, se preguntó a los sujetos sobre su estatus de no fumador o de } \\
\text { fumador y se tomaron medidas de CO. Se comentaron las habilidades que los sujetos habian } \\
\text { puesto en marcha para afrontar la situación de abstinentes y/o las circunstancias en que habian } \\
\text { recaido en el consumo de cigarrillos. } \\
\text { Además se agradeció a cada sujeto la participación en el programa. }\end{array}$ \\
\hline & & \\
\hline
\end{tabular}




\section{Procedimiento}

Cuando los sujetos solicitaban participar en el programa, se les entregaba un ejemplar del Cuestionario de información preliminar con el propósito de que fuera cumplimentado antes de acudir a la primera sesión de tratamiento. También en ese momento, se les entregaban hojas para los auto-registros, y se les explicaba, brevemente, cómo deblan de cumplimentarlas durante los siete dias previos al inicio del programa, con el fin de establecer la línea base de consumo de cigarrillos.

Los sujetos seleccionados se encontraban divididos en cuatro grupos en función de su ubicación geográfica y laboral. Mediante un sorteo, dos de estos grupos fueron asignados al tratamiento RGT, y los otros dos al tratamiento DHF. En total 20 sujetos fueron sometidos al tratamiento RGT y 22 sujetos al Tratamiento DHF. El tratamiento fue aplicado de manera colectiva para cada uno de estos grupos, aunque respetando las necesidades individuales de cada sujeto.

Las sesiones de tratamiento se llevaron a cabo una vez a la semana, de forma ininterrumpida durante dos meses, con un total de ocho sesiones presenciales y los correspondientes periodos intersesiones en los que los sujetos debian realizar una serie de tareas encomendadas por el terapeuta; la duración de cada sesión de tratamiento era de 90 minutos aproximadamente.

Tal y como puede observarse en la tabla 4, el esquema de desarrollo de las sesiones fue el mismo para los dos tipos de tratamiento; básicamente, cada sesión se dividió en tres fases, dedicadas principalmente a: (1) revisión de tareas realizadas en el periodo inter-sesiones anterior; (2) actividades centrales de la sesión; y (3) asignación de tareas para el siguiente periodo inter-sesiones. Sin embargo, cada tratamiento fue aplicado teniendo en cuenta sus peculiaridades.

El seguimiento a los tres y a los seis meses de finalizado el tratamiento, se realizó por medio de contactos telefónicos entre el terapeuta y los sujetos; y a los doce meses, tuvo lugar una entrevista individual con cada uno de los participantes y, posteriormente, una reunión con cada grupo. Todos los sujetos conocian la fecha aproximada en la que el terapeuta se pondría en contacto con ellos; y tenian la consigna de que si en algún momento precisaban comentar algún aspecto relacionado con la evolución de su situación, podian telefonearle para consultar sus dudas.

\section{Análisis de los datos}

Para analizar los datos, los dos grupos de sujetos sometidos al mismo tratamiento, fueron considerados un mismo grupo; contemplándose, por tanto dos grupos: el grupo RGT y el grupo DHF. En ambos grupos se realizaron comparaciones intra-grupo en cada una de la variables dependientes, mediante la prueba no paramétrica para muestras relacionadas de Wilcoxon cuando se compararon puntuaciones medias y la prueba de $\chi^{2}$ cuando se compararon números de sujetos.

Asimismo, se realizaron comparaciones entre los dos grupos de tratamientos en todas las variables dependientes, en este caso, empleando la prueba no paramétrica de la $U$ de Mann-Whitney cuando se consideraron puntuaciones medias, la prueba de $\chi^{2}$ cuando se compararon número de sujetos, y el contraste de hipótesis para dos proporciones en las comparaciones entre porcentajes.

\section{RESULTADOS \\ Variables intervinientes}

Como puede apreciarse en la tabla 5, ninguna diferencia entre los dos grupos de tratamiento, es estadisticamente significativa en las variables consideradas.

\section{Tasas de abstinencia}

En las tablas 6 y 7, aparecen los datos que corresponden, respectivamente, a los grupos RGT y DHF. En la figura 1, puede observarse la comparación de los datos de ambos grupos; y en la tabla 8, se presentan y comparan los porcentajes de sujetos abstinentes en las dos condiciones de tratamiento, al finalizar la intervención y en los correspondientes seguimientos. Como puede apreciarse en estas tablas y en esta figura, el tratamiento DHF propició un indice de abstinencia más elevado que el tratamiento RGT, aunque en ambos casos, hubo recaidas a lo largo de los seguimientos. Cabe destacar que el $86,6 \%$ de los sujetos tratados 
Tabla 5.- Puntuaciones medias (y DT) obtenidas por los sujetos en cada condición de tratamiento, en las variables intervinientes; se presentan también los valores $t$ de la comparación entre las medias de los sujetos de cada condición

\begin{tabular}{|c|c|c|c|}
\hline \multirow[b]{2}{*}{ Variables } & Condiciones & Tratamiento & \multirow[b]{2}{*}{$\mathbf{t}$} \\
\hline & $\begin{array}{l}\text { DHF } \\
N=22\end{array}$ & $\begin{array}{l}\text { RGT } \\
N=20\end{array}$ & \\
\hline Edad & $41,18(7,39)$ & $39.4(8.78)$ & 0.713 \\
\hline Años como fumador habitual & $23,38(7,41)$ & $19,05(8,32)$ & 1,739 \\
\hline Duración de abstinencia previa (semanas) & $15,25(21,48)$ & $25,13(42,23)$ & $-0,941$ \\
\hline $\begin{array}{l}\text { Cuánto pagaría para dejar de fumar } \\
\text { (\% de sueldo mensual) }\end{array}$ & $9,95(17,08)$ & $10,65(15,94)$ & $-0,130$ \\
\hline Ayuda que cree necesitar $(0-10)$ & $8,90(1,30)$ & $8,63(1,92)$ & 0,521 \\
\hline Ganas de dejar de fumar $(0-10)$ & $8,47(1,63)$ & $8.47(2,11)$ & 0,004 \\
\hline Esfuerzo cree será necesario $(0-10)$ & $9,09(1,22)$ & $9,15(1,70)$ & $-0,134$ \\
\hline Desednecesidad de fumar (Fagerstrón) & $4,42(2,19)$ & $4,21(2,38)$ & 0,295 \\
\hline Consumo estimado & $26(10,004)$ & $27,4(13,74)$ & $-0,379$ \\
\hline Consumo medido en linea base & $22,40(7,90)$ & $22,85(10,32)$ & $-0,156$ \\
\hline $\begin{array}{l}\text { Monóxido de carbono medido en L.B. } \\
\text { (partes por millón) }\end{array}$ & $39,68(16,18)$ & $36,9(13,99)$ & 0,593 \\
\hline
\end{tabular}

Tabla 6.- Número de sujetos tratados con el programa RGT, que estaban abstinentes en los diferentes momentos del estudio; y consumo medio de cigarrillos en la línea base, al final del tratamiento $y$ en los seguimientos a los tres, seis $y$ doce meses

\begin{tabular}{|c|c|c|c|c|c|}
\hline \multirow[b]{2}{*}{$\begin{array}{l}\text { Momento de la } \\
\text { observación }\end{array}$} & \multicolumn{5}{|c|}{$\begin{array}{l}\text { Consumo de cigarrillos } \\
\text { Medias (DT) }\end{array}$} \\
\hline & $\begin{array}{l}N^{0} \text { de sujetos } \\
\text { abstinentes } \\
(\%)\end{array}$ & $\begin{array}{l}\text { Sujetos que } \\
\text { no finalizaron } \\
(\mathrm{N}=3)\end{array}$ & $\begin{array}{l}\text { Sujetos que } \\
\text { finalizaron } \\
(\mathrm{N}=17)\end{array}$ & $\begin{array}{l}\text { Sujetos que } \\
\text { finalizaron y } \\
\text { no cesaron } \\
(\mathrm{N}=8)\end{array}$ & $\begin{array}{l}\text { Sujetos que } \\
\text { finalizaron y } \\
\text { cesaron } \\
(\mathrm{N}=9)\end{array}$ \\
\hline $\begin{array}{l}\text { Consumo estimado } \\
\text { en linea base }\end{array}$ & 0 & $38,66(25,1)$ & $27,4 \quad(13,74)$ & $30(8,68)$ & $21,33(11,37)$ \\
\hline $\begin{array}{l}\text { Consumo registrado } \\
\text { en linea base }\end{array}$ & 0 & $37(10,8)$ & $22,85(10,32)$ & $23,87(6,10)$ & $21,12(8,23)$ \\
\hline Sesión final & $9(45 \%)$ & & $4,66(5,56)$ & $11(7,25)$ & 0,00 \\
\hline $\begin{array}{l}\text { Seguimiento } \\
3 \text { meses }\end{array}$ & $5(25 \%)$ & & $9,33(7,88)$ & $17,12(4,18)$ & $3,11(5,55)$ \\
\hline $\begin{array}{l}\text { Seguimiento } \\
6 \text { meses }\end{array}$ & $3(15 \%)$ & & $12,73(7,03)$ & $18,25(4,26)$ & $7,77(7,18)$ \\
\hline $\begin{array}{l}\text { Seguimiento } \\
12 \text { meses }\end{array}$ & $2(15 \%)$ & $27,33(6,42)$ & $16,20(8,79)$ & $17,75(6,25)$ & $11,11(7,81)$ \\
\hline
\end{tabular}


Tabla 7.- Número de sujetos tratados con el programa DHF, que estaban abstinentes en los diferentes momentos del estudio; y consumo medio (y DT) de cigarrillos en la línea base, al final del tratamiento y en los seguimientos a los tres, seis y doce meses

\begin{tabular}{|c|c|c|c|c|c|}
\hline \multirow[b]{2}{*}{$\begin{array}{l}\text { Momento de la } \\
\text { observación }\end{array}$} & \multicolumn{5}{|c|}{$\begin{array}{l}\text { Consumo de cigarrillos } \\
\text { Medias (DT) }\end{array}$} \\
\hline & $\begin{array}{l}N^{0} \text { de sujetos } \\
\text { abstinentes } \\
(\%)\end{array}$ & $\begin{array}{l}\text { Sujetos que } \\
\text { no finalizaron } \\
(N=7)\end{array}$ & $\begin{array}{l}\text { Sujetos que } \\
\text { finalizaron } \\
(N=15)\end{array}$ & $\begin{array}{l}\text { Sujetos que } \\
\text { finalizaron y } \\
\text { no cesaron } \\
(\mathrm{N}=2)\end{array}$ & $\begin{array}{l}\text { Sujotos que } \\
\text { finalizaron y } \\
\text { cesaron } \\
(\mathrm{N}=13)\end{array}$ \\
\hline $\begin{array}{l}\text { Consumo estimado } \\
\text { en línea base }\end{array}$ & 0 & $25,71(12,39)$ & $26(10)$ & $35(7,07)$ & $24,76(8,88)$ \\
\hline $\begin{array}{l}\text { Consumo registrado } \\
\text { en linea base }\end{array}$ & 0 & $21,28(7,73)$ & $22,40(7,9)$ & $25,5(0,7)$ & $22,53(8,77)$ \\
\hline Sesión final & $13(59,1 \%)$ & $\cdots$ & $2,62(5,96)$ & $4(5,65)$ & 0.00 \\
\hline $\begin{array}{l}\text { Seguimiento } \\
3 \text { meses }\end{array}$ & $10(45,4 \%)$ & - & $11,04(10,46)$ & $20 \quad(7,07)$ & $4,69(7,44)$ \\
\hline $\begin{array}{l}\text { Seguimiento } \\
6 \text { meses }\end{array}$ & $8(36,3 \%)$ & - & $12,50(10,56)$ & $22,5 \quad 3,53)$ & $6,76 \quad(9)$ \\
\hline $\begin{array}{l}\text { Seguimiento } \\
12 \text { meses }\end{array}$ & $7(27,2 \%)$ & $20,57 \quad(7,91)$ & $13,90 \quad(10,9)$ & $21,5 \quad(4,9)$ & $9,15(10,84)$ \\
\hline
\end{tabular}

con el tratamiento DHF, que finalizaron el tratamiento (frente al $52,9 \%$ del grupo RGT), 10graron abandonar el consumo de tabaco, $y$ que de éstos, el $53 \%$ (frente al $22,2 \%$ del grupo RGT) se mantenia abstinente un año después de la intervención.

\section{Consumo diario de cigarrillos}

En las tablas 6 y 7 , también pueden contemplarse los datos correspondientes a esta variable dependiente. En los dos grupos, las únicas reducciones estadisticamente significativas en el seguimiento realizado a los doce meses, se produjeron en los sujetos que finalizaron el tratamiento; en el caso del grupo RGT, con independencia de que hubiesen abandonado el hábito al finalizar el tratamiento, mientras que en el grupo DHF, sólo en el subgrupo de sujetos que no fumaban al finalizar la intervención. No se encontraron diferencias estadisticamente significativas entre ambos grupos.
Semanas de abstinencla durante el año posterior a la intervención

Considerando, únicamente, a los sujetos que finalizaron el tratamiento, la media de semanas de abstinencia del grupo sometido al tratamiento DHF (Media= 28,87; DT $=21,41$ ) fue claramente superior a la media de semanas de abstinencia del grupo tratado mediante el método RGT (Media= 10,43; $D T=17,2$ ), siendo la diferencia inter-grupos estadisticamente significativa $(p<0,01)$.

\section{Nivel de monóxido de carbono (CO) contenido en el alre expirado}

En la tabla 9, se encuentran los datos que corresponden a las partes por millón de monóxido de carbono en el aire expirado. Las reducciones estadisticamente significativas en ambos grupos, son consistentes con los datos observados respecto a las tasas de abstinencia. Las diferencias entre los dos grupos no son significativas. 


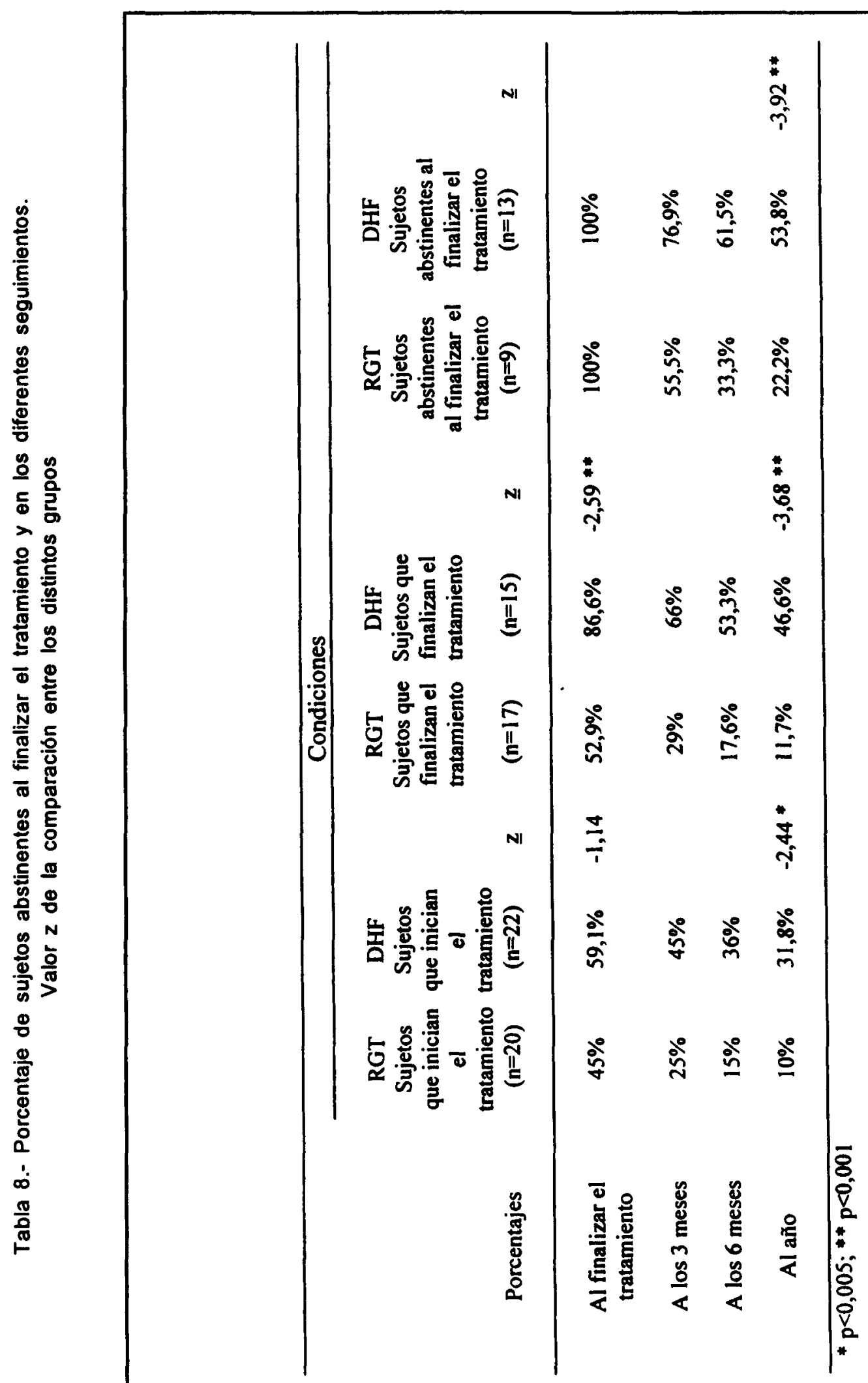




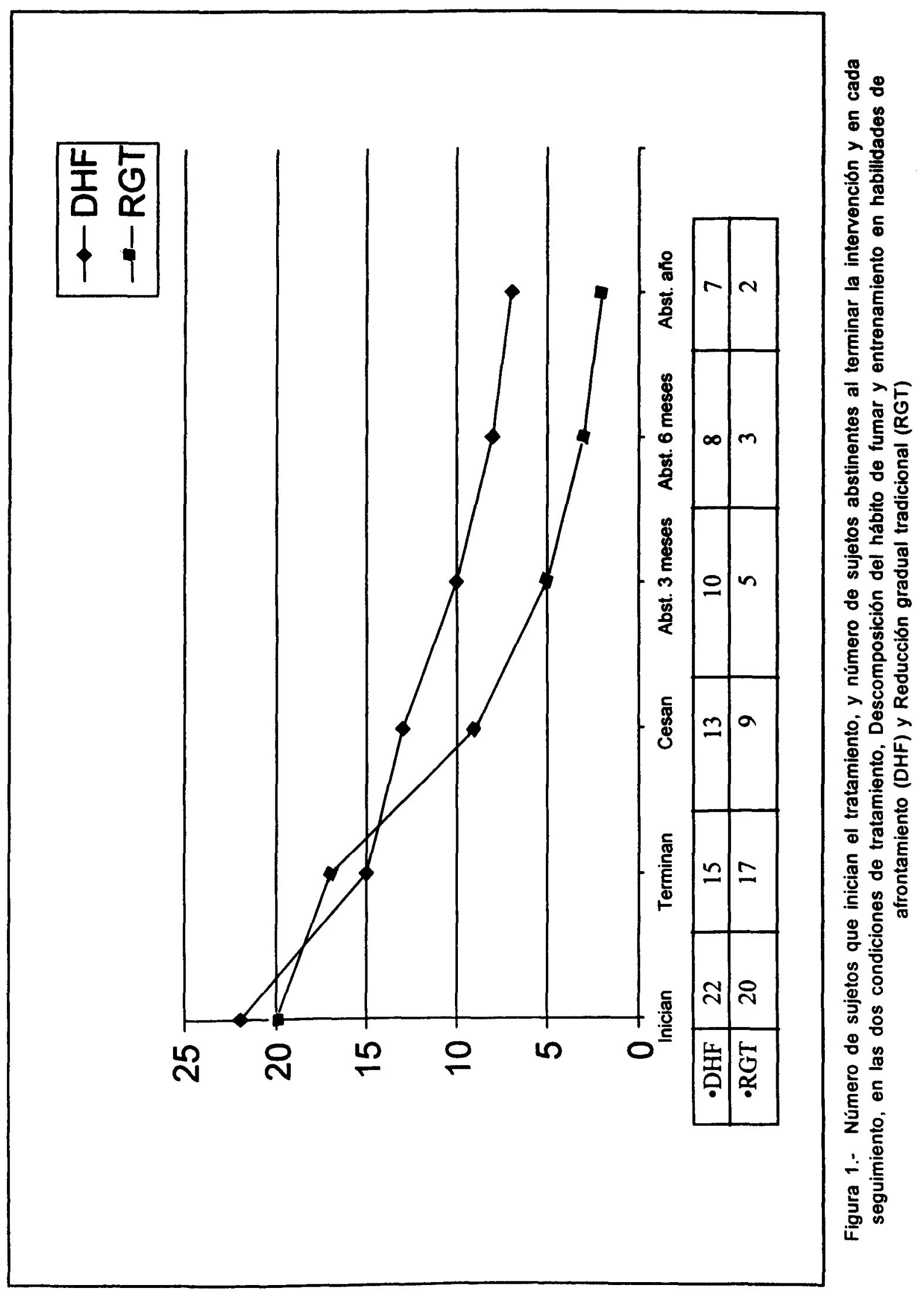


Tabla 9.- Puntuaciones medias (y DT) de partes por millón (ppm) de monóxido de carbono (CO) en el aire expirado según las mediciones realizadas en la línea base, al finalizar el tratamiento y en el seguimiento a los doce meses

\begin{tabular}{|c|c|c|c|c|c|}
\hline \multirow[b]{2}{*}{ Tratamiento } & \multirow[b]{2}{*}{$\begin{array}{l}\text { Momento de la } \\
\text { observación }\end{array}$} & \multicolumn{4}{|c|}{ Puntuación media de $\mathrm{CO}$} \\
\hline & & $\begin{array}{l}\text { Sujetos que } \\
\text { no finalizaron }\end{array}$ & $\begin{array}{l}\text { Sujetos que } \\
\text { finalizaron }\end{array}$ & $\begin{array}{l}\text { Sujetos que } \\
\text { finalizaron y } \\
\text { no cesaron }\end{array}$ & $\begin{array}{l}\text { Sujetos que } \\
\text { finalizaron y } \\
\text { cesaron }\end{array}$ \\
\hline \multirow[t]{3}{*}{ RGT } & Linea base & $\begin{array}{l}41,33 \\
(22,36)\end{array}$ & $\begin{array}{l}36,9 \\
(13,99)\end{array}$ & $\begin{array}{l}36,62 \\
(12,95)\end{array}$ & $\begin{array}{l}35,66 \\
(13,52)\end{array}$ \\
\hline & Sesión final & - & $\begin{array}{l}15,5 \\
(12,68)\end{array}$ & $\begin{array}{l}18,5 \\
(8,07)\end{array}$ & $\begin{array}{l}5,8 \\
(3,1)\end{array}$ \\
\hline & $\begin{array}{l}\text { Seguimiento } \\
12 \text { meses }\end{array}$ & $\begin{array}{l}38,66 \\
(14,43)\end{array}$ & $\begin{array}{l}26 \\
(11,51)\end{array}$ & $\begin{array}{l}28,62 \\
(8,46)\end{array}$ & $\begin{array}{l}19,44 \\
(9,25)\end{array}$ \\
\hline \multirow[t]{3}{*}{ DHF } & Línea base & $\begin{array}{l}44,85 \\
(17,53)\end{array}$ & $\begin{array}{l}39,68 \\
(16,18)\end{array}$ & $\begin{array}{l}56,5 \\
(31,81)\end{array}$ & $\begin{array}{l}35,92 \\
(12,45)\end{array}$ \\
\hline & Sesión final & -- & $\begin{array}{l}14,31 \\
(12,19)\end{array}$ & $\begin{array}{l}16 \\
(9,89)\end{array}$ & $\begin{array}{l}6,2 \\
(2,7)\end{array}$ \\
\hline & $\begin{array}{l}\text { Seguimiento } \\
12 \text { meses }\end{array}$ & $\begin{array}{l}31,76 \\
(14,43)\end{array}$ & $\begin{array}{l}20,77 \\
(11,77)\end{array}$ & $\begin{array}{l}26,5 \\
(4,94)\end{array}$ & $\begin{array}{l}15,84 \\
(12,08)\end{array}$ \\
\hline
\end{tabular}

Nota: RGT= Reducción gradual tradicional; $\mathrm{DHF}=$ Descomposición del hábito de fumar $y$ entrenamiento en habilidades de afrontamiento.

\section{DISCUSIÓN}

En el presente estudio se han comparado dos tratamientos cognitivo-conductuales de reducción gradual del consumo de cigarrillos: uno de corte más tradicional, basado en la reducción progresiva indiscriminada, según objetivos de disminución semanal (tratamiento RGT), y otro basado en la reducción gradual pero como consecuencia inevitable del proceso de descomposición del hábito de fumar (tratamiento DHF); este último método, además, ha incluido el entrenamiento de los sujetos en habilidades de afrontamiento que les permitiesen manejar las situaciones de riesgo una vez alcanzada la abstinencia. Los resultados de la investigación, señalan la superioridad del método DHF sobre el más tradicional RGT, tanto al finalizar el tratamiento como en los diferentes seguimientos a los tres, seis y doce meses.

Los datos observados en el grupo sometido al tratamiento DHF, coinciden con los obtenidos por Buceta y López de la Llave (1989) en un estudio anterior $(86,6 \%$ vs. $84,6 \%$ de sujetos abstinentes al finalizar la intervención; y $53,3 \%$ vs. $53,8 \%$ a los seis meses de seguimiento), pero además, en esta investigación, se ha podido comprobar la eficacia de este método de tratamiento, en comparación con un método de reducción gradual más tradicional, y se ha realizado un seguimiento a los doce meses, ausente en el estudio previo, que ha confirmado el mantenimiento de los logros terapéuticos en un considerable porcentaje de sujetos $(46,6 \%)$, claramente superior al del otro tratamiento $(11,7 \%)$.

La superioridad mostrada por el tratamiento DHF, no puede atribuirse a los componentes comunes a ambos programas de tratamiento (número de sesiones, explicaciones sobre el tabaco y sus efectos, atención terapéutica, autoregistros, otras técnicas comunes), sino a los particulares de este método; en concreto: la intervención específicamente centrada en el debilitamiento de las relaciones funcionales entre estimulos antecedentes, conducta y consecuencias, el control de cogniciones perjudiciales, y la preparación de los sujetos tanto para el momento estresante de la cesación (mediante 
las pruebas de cesación y abstinencia) como para el afrontamiento de las situaciones de riesgo una vez alcanzada la abstinencia. Estos elementos, que diferencian a los dos métodos de tratamiento, podrian ser responsables del mayor número de sujetos que han abandonado el consumo al finalizar el tratamiento, $y$ del mayor número que siguen sin consumir tabaco al año de seguimiento. Sin embargo, este estudio no 'permite discriminar el peso específico de cada elemento del tratamiento DHF, tarea que en el futuro deberia abordar otras investigaciones.

Las tasas de abstinencia conseguidas por el grupo DHF, aunque prometedoras, teniendo en cuenta las tasas bajas que habitualmente se observan en el ámbito del tratamiento del tabaquismo, señalan que el método empleado no ha resultado útil para muchos de los sujetos que han sido sometidos a esta intervención: en algunos casos, no se ha logrado el objetivo inicial de la cesación al finalizar el tratamiento, y en otros, no se ha conseguido el objetivo de mantener la abstinencia al año de seguimiento. Estos datos, sugieren, en primer lugar, que este método, y/o su aplicación en grupo, no son necesariamente útiles para todos los fumadores, debiendo encontrarse las caracteristicas individuales que aconsejen usarlos o desestimarlos; en segundo lugar, que, probablemente, deban estudiarse más a fondo sus distintos componentes, con el propósito de perfeccionarlo y mejorar su eficacia; en tercer lugar, que quizá sea conveniente prolongar la duración del tratamiento, añadiendo más sesiones y/o incorporando sesiones cuya frecuencia se vaya distanciando progresivamente (por ejemplo: cada quince dias inicialmente, mensuales en una segunda fase, bimensuales más adelante, etc.).

Los dos métodos empleados, han mostrado su eficacia para reducir el consumo de cigarrillos en aquellos sujetos que no han abandonado el hábito. Fumar poco, puede resultar muy reforzante y contribuir, así, a dificultar la eliminación definitiva del hábito, aumentando la probabilidad de que se incremente la tasa de consumo cuando el sujeto se enfrente a situaciones especialmente estresantes, y se mantenga elevada a partir de entonces. Sin embar- go, los riesgos para la salud son menores si se fuma menos, y para muchas personas con una adicción muy consolidada, parece que ésta es la única alternativa viable al consumo más perjudicial; lo que sugiere que los programas de tratamiento en este ámbito, puesto que en la mayoría de los casos contribuyen a una reducción sustancial del número de cigarrillos, deberian incluir estrategias especificas que ayudasen a los participantes que no consiguen la abstinencia total, a mantenerse en una tasa de consumo baja. En concreto, podrian anticiparse las situaciones de mayor riesgo, y entrenar a los sujetos para controlarlas mediante conductas alternativas, que fuesen más saludables que el consumo de tabaco.

En definitiva, el método de reducción gradual basado en la descomposición del hábito y la preparación del fumador para la abstinencia, constituye una alternativa prometedora en el ámbito del tratamiento del tabaquismo, pero nuevas investigaciones, partiendo de las conclusiones que han sido senaladas en este trabajo, deben aportar más datos.

\section{REFERENCIAS BIBLIOGRAFICAS}

American Psychiatric Association (1995). Manual diagnóstico y estadistico de los trastornos mentales (DSM IV). Barcelona: Masson

Becona, E. (1987). Intervención psicológica para la eliminación del hábito de fumar. En J.M.Buceta (Ed.) Psicología clinica y salud: Aplicación de estrategias de intervención. 257-282. Madrid: UNED editorial.

Becona, E. ; Galego, P. y Lorenzo, M.C. (1988). EI tabaco y su abandono. Santiago de Compostela: Dirección Xeral de Saúde Pública da Consellería de Sanidades da Xunta de Galicia

Buceta, J.M. y López de la Llave, A. (1989). Aplicación de un programa de intervención cognitivocomportamental para la cesación del comportamiento de fumar. Revista Intercontinental de Psicología y Educación. 2, 81-99.

Buceta, J.M. y Thomas, M. (1983). Behavioural treatment of smoking: Some suggestions from clinical group experiences. Birch Hill Hospital, Rochdale, Inglaterra (no publicado)

Fagerström, K.O. (1978). Measuring degree of physical dependence to tobacco smoking with reference to individualization of treatment. Addictive Behaviors. 3, 235-241. 
Günther, V., Gritsch, S y Meise, U. (1992) Smoking cessation-gradual or sudden stopping?. Drugs and Alcohol Dependence, 29, 231-236

López de la Llave, A. y Buceta, J.M. (1996). Tratamiento psicológico del hábito de fumar. En J.M. Buceta y A.M. Bueno (Eds) Tratamiento psicológico de hábitos y enfermedades. 103162. Madrid. Pirámide.

Schwartz, J.L. (1987). Review and evaluation of smoking cessation methods. The United States and Canada, 1978-1985. Maryland: US Department of Health, Education and Welfare.

Shiffman, S., Read, L., Maltese, J., Rapkin, D. y Jarvik, M. E. (1985). Preventing relapse in exsmokers: A self-management approach. En Marlatt, G.A. y Gordon, J.R. (Eds.) Relapse Prevention. Maintenance Strategies in the Treatment of Addictive Behaviors. 472-520. New York: The Guilford Press. 